\title{
Development and Evaluation of CmeC Subunit Vaccine against Campylobacter jejuni
}

\author{
Ximin Zeng, Fuzhou Xu and Jun Lin*
}

Department of Animal Science, The University of Tennessee, Knoxville, TN 37996, USA

\begin{abstract}
Campylobacter jejuni is the leading bacterial cause of human enteritis in many industrialized countries. There is no commercial vaccine against $C$. jejuni available to date. $\mathrm{CmeC}$ is an essential outer membrane component of CmeABC multidrug efflux pump that plays a critical role in antibiotic resistance and in vivo colonization of $C$. jejuni. $\mathrm{CmeC}$ is prevalent in C. jejuni strains and is dramatically induced and immunogenic in vivo. In this study, we analyzed $\mathrm{CmeC}$ sequence homology, examined in vitro immune protection of $\mathrm{CmeC}$ peptide antibodies, and produced full-length recombinant $\mathrm{CmeC}(\mathrm{r} \mathrm{CmeC}$ ) for evaluating immunogenicity and protective efficacy of the $\mathrm{CmeC}$ subunit vaccine against $C$. jejuni using chicken model system. Amino acid sequences of $\mathrm{CmeC}$ from 24 diverse $C$. jejuni strains were determined and subjected to alignment, which revealed that $\mathrm{CmeC}$ is highly conserved in $C$. jejuni with a identity ranging from $97.3 \%$ to $100 \%$. CmeC peptide antibodies inhibited the function of CmeABC efflux pump and enhanced susceptibility of $C$. jejuni to bile salts, the natural antimicrobial present in the intestine. Two full-length $\mathrm{rCmeC}$ proteins with $\mathrm{N}$ - or C-terminal His tag were produced in $E$. coli; the $\mathrm{N}$-terminal His-tagged $\mathrm{rCmeC}$ with high purity and yield was obtained by single step affinity purification. The purified $\mathrm{rCmeC}$ was used in two vaccination trials using a chicken model of $C$. jejuni infection. Stimulation of $\mathrm{CmeC}$-specific serum IgG responses via oral vaccination required immunization with higher doses of $\mathrm{rCmeC}(200 \mu \mathrm{g})$ together with $70 \mu \mathrm{g}$ of mucosal adjuvant $\mathrm{mLT}$ (modified $E$. coli heat-labile enterotoxin). Subcutaneous vaccination of chickens with $\mathrm{rCmeC}$ remarkably stimulated both serum IgG and IgA responses. However, $\mathrm{CmeC}$-specific intestinal secretory IgA response was not significantly stimulated regardless of vaccination regimen and the $\mathrm{rCmeC}$ vaccination did not confer protection against $C$. jejuni infection. Together, these findings provide further compelling evidence that $\mathrm{CmeC}$ is a promising subunit vaccine candidate against $C$. jejuni infection. However, the $\mathrm{CmeC}$ vaccination regimen should be optimized to enhance $\mathrm{CmeC}$-specific mucosal immune response in the intestine for protection against $C$. jejuni.
\end{abstract}

Keywords: Campylobacter jejuni; Subunit vaccine; Multidrug efflux pump

\section{Introduction}

Campylobacter jejuni is the leading bacterial cause of human enteritis in the United States and other industrialized countries [1]. This pathogenic organism causes watery diarrhea and/ or hemorrhagic colitis in humans and is also associated with Guillain-Barré syndrome, an acute flaccid paralysis that may lead to respiratory muscle compromise and death $[2,3]$. Poultry are the major reservoir of Campylobacter and thus the main source for human campylobacteriosis $[1,4]$. At the same time that prevalence of infection is increasing, Campylobacter has become increasingly resistant to antibiotics, including fluoroquinolones and macrolides, the major drugs of choice for treating human campylobacteriosis [5]. Despite the growing need for new antibiotics due to increasing drug resistance in Campylobacter and other bacteria, many pharmaceutical companies have been placing less emphasis on antibiotic discovery [6]. Therefore, alternative intervention strategies, such as vaccination, are needed to prevent and control Campylobacter infections. To dates, vaccines against Campylobacter infection are still not available, primarily due to the antigenic complexity of this organism and a lack of understanding of the mechanisms of pathogenesis. Information concerning protective antigens as vaccine candidates in $C$. jejuni is limited and vaccinations against $C$. jejuni using animal models including chickens have had only partial success [7-9].

It has been well established that prior infection with $C$. jejuni can induce protective immunity against Campylobacter infections in humans and animals, strongly supporting the feasibility of development of immunization-based approaches to control Campylobacter infections [7]. Outer membrane proteins (OMPs) of $C$. jejuni are considered the major mediators of pathogenhost interactions and are promising candidates for the design of protective vaccines. Recently, we characterized a unique OMP CmeC, an essential component of multidrug efflux pump CmeABC that plays a critical role in antibiotic resistance and pathogenesis of $C$. jejuni [10-13]. The $\mathrm{CmeC}$ is a promising subunit vaccine candidate against C. jejuni because of following compelling evidences. First, $\mathrm{CmeC}$ is essential for $C$. jejuni colonization in animal intestine by mediating bile resistance $[10,11,13]$. Compared to the wild type strain that colonized the chickens as early as day 2 post-inoculation with a density as high as $10^{7} \mathrm{CFU} / \mathrm{g}$ feces, the isogenic $\mathrm{CmeC}$ mutant failed to colonize any of the inoculated chickens throughout the study [12]; the minimum infective dose for $\mathrm{CmeABC}$ mutant is at least $2.6 \times 10^{4}$ folds higher than that of the wild-type strain [12]. Second, PCR and immunoblotting analyses showed that $\mathrm{CmeC}$ is widely existed and constitutively expressed among different $C$. jejuni strains, suggesting that $\mathrm{CmeC}$ is highly conserved in terms of sequence and antigenicity [11]. Third, expression of $\mathrm{CmeC}$ is dramatically induced by bile salts present in the intestine, further highlighting the critical role of $\mathrm{CmeC}$

${ }^{*}$ Corresponding author: Dr. Jun Lin, Department of Animal Science, The University of Tennessee 2640 Morgan Circle Drive, Knoxville, TN 37996-4574 USA, Tel: (865)974-5598; Fax: (865)974-7297; E-mail: jlin6@utk.edu

Received November 30, 2010; Accepted December 30, 2010; Published December 31, 2010

Citation: Zeng X, Xu F, Lin J (2010) Development and Evaluation of CmeC Subunit Vaccine against Campylobacter jejuni. J Vaccines Vaccin 1: 112. doi:10.4172/21577560.1000112

Copyright: (c) 2010 Zeng X, et al. This is an open-access article distributed under the terms of the Creative Commons Attribution License, which permits unrestricted use, distribution, and reproduction in any medium, provided the original author and source are credited. 
Citation: Zeng X, Xu F, Lin J (2010) Development and Evaluation of CmeC Subunit Vaccine against Campylobacter jejuni. J Vaccines Vaccin 1: 112. doi:10.4172/2157-7560.1000112

Page 2 of 8

in Campylobacter pathogenesis [13]. This notion also is supported by a recent microarray study by Stintzi et al. [14], in which expression of cme $A B C$ operon was found to be highly up-regulated in vivo. In addition, using multiple chicken sera for immunoblot analysis, we also demonstrated that $\mathrm{CmeC}$ is expressed during Campylobacter infection of chickens and elicited a specific antibody response in the host [12], supporting the feasibility of targeting CmeC for immune protection against $C$. jejuni colonization. Finally, we also demonstrated that inhibition of $\mathrm{CmeABC}$ by efflux pump inhibitors increased susceptibility of Campylobacter to various antimicrobials, prevented emergence of macrolide resistant $C$. jejuni, and reduced in vivo colonization of $C$. jejuni using a chicken model system $[15,16]$. Based on these observations, we hypothesize that CmeC antibodies could inhibit functions of CmeABC pump and that $\mathrm{CmeC}$ is a promising subunit vaccine candidate to prevent and control $C$. jejuni colonization in the intestine.

In this study, we determined sequence homology of $\mathrm{CmeC}$ in diverse Campylobacter strains as well as in vitro immune protection of $\mathrm{CmeC}$ peptide antibodies, which further support the feasibility of targeting $\mathrm{CmeC}$ for immune intervention against $C$. jejuni infection. We also constructed plasmids for producing full-length $\mathrm{rCmeC}$ and optimized conditions for purification of large quantities of $\mathrm{rCmeC}$ with high purity. The purified $\mathrm{rCmeC}$ was then used in two large chicken vaccination trials to evaluate the immunogenicity and protective efficacy of the $\mathrm{CmeC}$ subunit vaccine with different vaccination regimens.

\section{Materials and Methods}

\section{Bacterial strains, plasmids and culture conditions}

The major bacterial strains and plasmids used in this study are listed in Table 1. Fourteen $C$. jejuni isolates (JL7, 10, 12, 36, 78, 81, 83, $85,90,91,93,94,95$ and 118) from various sources (human, chicken, turkey, bovine, and environment) were used for PCR amplification and sequence analysis of the complete $c m e C$ gene. The $C$. jejuni strains were routinely grown in Mueller Hinton (MH) broth (Difco, Detroit, MI) or on $\mathrm{MH}$ agar at $42^{\circ} \mathrm{C}$ under microaerophilic conditions, which were generated using CampyGen Plus (Oxoid, Bashingstoke, Hampshire, England) gas pack in an enclosed jar. Escherichia coli was grown in Luria-Bertani (LB) broth (Fisher Scientific, Fairlawn, NJ) with shaking $(250 \mathrm{rpm})$ or on agar at $37^{\circ} \mathrm{C}$ overnight. When needed, culture media were supplemented with ampicilin $(100 \mu \mathrm{g} / \mathrm{ml})$ (Sigma, St Louis, MO).

\section{PCR amplification and sequence analysis of the $\mathrm{CmeC}$ gene}

Genomic DNA was extracted from each isolate by using Wizard $^{\circledR}$ Genomic DNA Purification Kit (Promega, Madison, WI). Primers CmeCF (5'-CAGCAAAACTTCGTTTTCGTC-3') and CmeCR (5' TGCCTGCTATTTACAAGGCTTA-3') were used to amplify the entire cmeC gene. Amplified PCR products were purified by QIAquick PCR Purification Kit (Qiagen, Hilden, Germany) and sequenced in Molecular Biology Resource Facility at The University of Tennessee (Knoxville, $\mathrm{TN})$. The cmeC sequences were converted to amino acid sequences and subjected to alignment together with other publicly available CmeC sequences (Accession \#: ABC59229, AAT38952, CAL34515, YP_001000076, ZP_01100537, ZP_01069571, ZP_01067179, YP_178433, ZP_01071951) using DNAStar package (version 6.0). To predict the secondary structure of $\mathrm{CmeC}$, the amino acid sequence of $\mathrm{CmeC}$ was analyzed by using the program TransMembrane protein Re-Presentation in 2 Dimensions tool (TMRPres2D) [17]. Homology Modeling was performed using an automated model on SWISS Model Workspace (http://swissmodel.expasy.org/workspace/), using NodT (1wp1.pdb) as a template.

\section{Effect of $\mathrm{CmeC}$ peptide antibodies on susceptibility of $C$. jejuni to bile salts}

We have prepared a recombinant strain of E. coli JM109 expressing a portion of $\mathrm{CmeC}$ (aa 41 to 248 of total 492 aa in length) and high-titer of specific rabbit polyclonal antiserum directed against this $\mathrm{CmeC}$ peptide was also generated [11]. This $\mathrm{CmeC}$ peptide antiserum was used to examine the effect of $\mathrm{CmeC}$ peptide antibodies on susceptibility of $C$. jejuni 81-176 to cholic acid, a representative bile salt. Briefly, a log-phase culture of $C$. jejuni 81-

\begin{tabular}{|c|c|c|}
\hline Plasmids or Strains & Description & Source or Reference \\
\hline \multicolumn{3}{|l|}{ Plasmids } \\
\hline pGEMT-Easy & PCR cloning vector, $\mathrm{Amp}^{r}$ & Promega \\
\hline pQE-30 & Vector for $\mathrm{N}$-terminal His-tagged protein construction & Qiagen \\
\hline pQE-70 & Vector for C- terminal His-tagged protein construction & Qiagen \\
\hline pCmeC-NHIS & pQE-30 ligated with $\mathrm{cmeC}$ segment encoding mature $\mathrm{CmeC}$ & This study \\
\hline pCmeC-CHIS & pQE-70 ligated with $\mathrm{cmeC}$ segment encoding mature $\mathrm{CmeC}$ & This study \\
\hline \multicolumn{3}{|l|}{ Strains } \\
\hline \multicolumn{3}{|l|}{ C. jejuni } \\
\hline JL7 & H49024, human isolate & {$[29]$} \\
\hline JL10 & ATCC 33291, human isolate & ATCC \\
\hline $\mathrm{JL} 12$ & 15046764, bovine isolate & [29] \\
\hline JL36 & S3B, chicken isolate & {$[12]$} \\
\hline $\mathrm{JL} 78$ & W42606, human isolate & [42] \\
\hline JL81 & F34078, human isolate & {$[42]$} \\
\hline JL83 & M76297, human isolate & {$[42]$} \\
\hline JL85 & F59966, human isolate & {$[42]$} \\
\hline JL90 & M33323, human isolate & {$[42]$} \\
\hline JL91 & W11805, human isolate & [42] \\
\hline JL93 & M402, human isolate & {$[18]$} \\
\hline JL94 & E46972, human isolate & {$[42]$} \\
\hline JL95 & 19094451, ovine isolate & {$[29]$} \\
\hline JL118 & CVM20088, chicken isolate & [43] \\
\hline JL241 & NCTC 11168, human isolate & {$[44]$} \\
\hline JL242 & $81-176$, human isolate & [45] \\
\hline \multicolumn{3}{|l|}{ E. coli } \\
\hline $\mathrm{DH} 5 \alpha$ & F- Ф80lacZ $\Delta$ M15 $\Delta$ (lacZYA-argF)U169 recA1 endA1 hsdR17 (rk-, mk+) phoA supE44 thi-1 gyrA96 relA1 $\lambda-$ & Invitrogen \\
\hline JM109 & endA1 recA1 gyrA96 thi hsdR17 (rk-, mk+) relA1 supE44 $\Delta$ (lac-proAB) [F' traD36 laclqZ $\Delta \mathrm{M} 15]$ & Promega \\
\hline JL243 & JM109 containing pCmeC-NHIS & This study \\
\hline JL348 & JM109 containing pCmeC-CHIS & This study \\
\hline
\end{tabular}

Table 1: Key bacterial strains and plasmids used in this project. 


\begin{tabular}{|c|c|c|c|c|c|}
\hline Group & No. of Chickens & Day 7 Primary Immunization & Day 21 Booster Immunization & Sample Collections & C. jejuni challenge on day 35 \\
\hline 1 & 20 & \multicolumn{2}{|l|}{ PBS } & \multirow{6}{*}{$\begin{array}{l}\text { Blood: day } 7 \\
21,35,42 \\
\text { Intestinal } \\
\text { lavage: day } \\
21,35\end{array}$} & Yes \\
\hline 2 & 20 & $\mathrm{mLT}^{\mathrm{a}}$ & & & Yes \\
\hline 3 & 20 & CmeC $(50 \mu g)$ & & & Yes \\
\hline 4 & 20 & CmeC $(50 \mu \mathrm{g})+\mathrm{mLT}(10 \mu \mathrm{g})$ & & & Yes \\
\hline 5 & 20 & $\mathrm{CmeC}(200 \mu \mathrm{g})$ & & & Yes \\
\hline 6 & 20 & $\mathrm{CmeC}(200 \mu \mathrm{g})+\mathrm{mLT}(10 \mu \mathrm{g})$ & & & Yes \\
\hline
\end{tabular}

amLT: mucosal adjuvant LT-R192G, a mutant E. coli heat-labile toxin kindly provided by Dr. John D. Clements (Tulane University Medical Center).

Table 2: The experimental design of the first $\mathrm{CmeC}$ vaccination and $C$. jejuni challenge using broilers (Trial 1).

\begin{tabular}{|c|c|c|c|c|c|c|}
\hline Group & No. of Chickens & Vaccination Route & Day 21 Primary Immunization & Day 35 Booster Immunization & \multirow{7}{*}{$\begin{array}{l}\text { Samplood: day } 21,35,49, \\
63 ; \text { Intestinal lavage: } \\
\text { day } 35,49 \text {; Cloacal } \\
\text { swab: day } 49,51,53 \text {, } \\
56,58 .\end{array}$} & C. jejuni challenge on day 49 \\
\hline A & 20 & Oral & \multicolumn{2}{|l|}{$\mathrm{mLT}^{\mathrm{a}}(70 \mu \mathrm{g})$} & & Yes \\
\hline B & 20 & Oral & \multicolumn{2}{|l|}{ CmeC $(200 \mu \mathrm{g})$} & & Yes \\
\hline $\mathrm{C}$ & 20 & Oral & \multirow{2}{*}{\multicolumn{2}{|c|}{\begin{tabular}{|l|} 
CmeC $(50 \mu \mathrm{g})+\mathrm{mLT}(70 \mu \mathrm{g})$ \\
CmeC $(200 \mu \mathrm{g})+\mathrm{mLT}(70 \mu \mathrm{g})$
\end{tabular}}} & & Yes \\
\hline $\mathrm{D}$ & 20 & Oral & & & & Yes \\
\hline $\mathrm{E}$ & 20 & Subcutaneous & \multicolumn{2}{|l|}{ FIAb } & & Yes \\
\hline $\mathrm{F}$ & 20 & Subcutaneous & \multicolumn{2}{|l|}{$\mathrm{CmeC}(200 \mu \mathrm{g})+\mathrm{FIAb}$} & & Yes \\
\hline
\end{tabular}

${ }^{\mathrm{a}} \mathrm{mLT}$ : mucosal adjuvant LT-R192G, a mutant $E$. coli heat-labile toxin kindly provided by Dr. John D. Clements (Tulane University Medical Center). ${ }^{\mathrm{b}} \mathrm{FIA}$ : Freund's incomplete adjuvant

Table 3: Experimental design of the second $\mathrm{CmeC}$ vaccination and $C$. jejuni challenge using white leghorns (Trial 2).

176 was diluted to approximately $2 \times 10^{6}$ colony forming unit (CFU) per milliliter in $\mathrm{MH}$ broth containing sublethal concentration of cholic acid $(2 \mathrm{mg} / \mathrm{ml})$. The anti-CmeC and control sera were added to cells with 1:10 dilution and cells were incubated for $6 \mathrm{hr}$ at $42^{\circ} \mathrm{C}$ under microaerophilic conditions. Samples were diluted serially in $\mathrm{MH}$ and plated on $\mathrm{MH}$ agar plates to determine bacterial viability. Both sera were inactivated at $56^{\circ} \mathrm{C}$ for $30 \mathrm{~min}$ prior to use to abolish complement activity. Two independent experiments were performed with triplicate assays in each experiment.

\section{Production and purification of full-length $\mathrm{rCmeC}$}

To construct recombinant strain producing full-length $\mathrm{N}$-terminal His-tagged $\mathrm{rCmeC}$, a 1,444-bp fragment encoding mature 473-aa $\mathrm{CmeC}$ (aa 20 to 492) with the removal of the 19 aa signal peptide was PCR amplified from $C$. jejuni NCTC 11168 using primers CCF (5'AAAGGATCCTGCTCTTTAAGTCCAAATTTAAATATT-3') and CCR (5'-AA ACCCGGGCTATTCTCTAAAAGACATATCTAAATT-3). The restriction sites (BamHI and Smal, underlined in the primer sequences) were attached to the 5' end of each primer to facilitate directional cloning of the amplified PCR product into the PQE30 vector (Qiagen, Hilden, Germany). The amplified $c m e C$ fragment was digested with BamHI and Smal and was cloned into the vector pQE-30, which previously had been digested with BamHI and Smal, creating pCmeC-NHIS (Table 1). Cloning, expression, and purification of $\mathrm{N}$-terminal His-tagged $\mathrm{rCmeC}$ were performed using the procedures described previously $[10,12,18]$. To construct a recombinant strain producing the full-length $\mathrm{C}$-terminal His-tagged $\mathrm{rCmeC}$, a 1,472-bp fragment encoding full length of 492-aa $\mathrm{CmeC}$ (aa 1 to 492) was PCR amplified from $C$. jejuni NCTC 11168 using primers CmeC_FLF (5'-ACATGCATGCATAAAATAATTTCAATTAGTG-3', Sphl site underlined) and CmeC FLR (5'- CCTAGATCTTTCTCTAAAAGACATATCTAAA-3', $B g$ III site underlined). The PCR amplified cmeC fragment was digested with $S p h \mathrm{I}$ and BgIII and was cloned into the pQE-70 (Qiagen, Hilden, Germany), which previously had been digested with the same enzymes, creating pCmeC-NHIS (Table 1). The other procedures are the same as above for purifying $\mathrm{N}$-terminal His-tagged rCmeC. Plasmids pCmeC-NHIS and pCmeC-CHIS were sequenced, with no frameshift and mutations in the coding sequence of $\mathrm{cmeC}$ detected. To optimize conditions of producing high-purity $\mathrm{rCmeC}$ for vaccination, $5 \mathrm{mM}$ of ATP- $\mathrm{Mg}^{2+}$ was added in lysis buffer to remove contamination of molecule chaperone GroEL [19] and 0.1\% Empigen BB (Sigma, St Louis, MO) was used to facilitate solubilization of rCmeC and preserve the antigenicity of $\mathrm{rCmeC}[10,12,18]$. The $\mathrm{N}$-terminal His-tagged $\mathrm{rCmeC}$ with high purity was used for vaccination trials described below.

\section{CmeC vaccination and $C$. jejuni challenge: Trial 1}

The experimental design is detailed in Table 2. Briefly, newly hatched broiler chickens $(n=120)$ were allocated randomly into 6 groups (20 per group). Each group of chickens were maintained in a sanitized wire-floored cage and provided with unlimited access to water and commercial chicken starter feed without antibiotic additives. At 1-week-old age, chickens were immunized orally with $200 \mu \mathrm{l}$ of $\mathrm{CmeC}$ vaccine in PBS via oral gavage. CmeC doses used were 50 and $200 \mu \mathrm{g}$, either alone or coadministered with $10 \mu \mathrm{g}$ of mLT (general gift from Dr. John Clements in Tulane University Medical Center). Chickens receiving PBS only or mLT only were used as control groups. Booster doses were administered 2 wk after primary immunization. Blood samples were collected from the wing vein of each chicken at 1, 3, 5, $7 \mathrm{wk}$ of the experiment to monitor circulating $\mathrm{CmeC}$ specific $\operatorname{IgA}$ and $\operatorname{IgG}$ antibodies; intestinal lavage samples were collected and prepared at 3 , and $5 \mathrm{wk}$ from euthanized chickens (5 bird/group) as described previously [20] to monitor mucosal IgA antibody response. Prior to challenge, chickens were screened again with conventional culture methods to ensure that they were Campylobacter-free. At week 5, 100 $\mu$ l culture of $C$. jejuni NCTC 11168 was inoculated orally into broilers $\left(10^{6} \mathrm{CFU} / \mathrm{bird}\right)$. After challenge, cloacal swabs were collected every $2 \mathrm{~d}$ for $10 \mathrm{~d}$. Swabs were diluted serially, and plated on $\mathrm{MH}$ agar containing selective supplement (SR117E; Oxoid, Bashingstoke, Hampshire, England) for enumeration of Campylobacter cells. The randomly selected colonies were tested by PCR [12] to ensure that output Campylobacter was the same as the inoculum and there were no contamination of chickens by other sources.

\section{CmeC vaccination and $C$. jejuni challenge: Trial 2}

The second vaccination trial (Table 3 ) had four major modifications compared to Trial 1(Table 2). First, white leghorn chickens, which grow slower than broilers, were used in this trial. Second, to compare effects of vaccination route on immune response and protective efficacy of $\mathrm{rCmeC}$, subcutaneous administration was included in this trial. Third, the dose of mucosal adjuvant mLT was increased from 10 to70 $\mu \mathrm{g} / \mathrm{chicken}$. Finally, the dose of $C$. jejuni NCTC 11168 used for challenge was reduced to $10^{5} \mathrm{CFU} / \mathrm{bird}$.

\section{Enzyme-linked immunoabsorbant assay (ELISA)}

CmeC-specific antibodies in intestinal lavage samples and sera were measured by indirect ELISA, which was performed using a previously published protocol [21] with the following modifications. 
Citation: Zeng X, Xu F, Lin J (2010) Development and Evaluation of CmeC Subunit Vaccine against Campylobacter jejuni. J Vaccines Vaccin 1: 112. doi:10.4172/2157-7560.1000112

Page 4 of 8

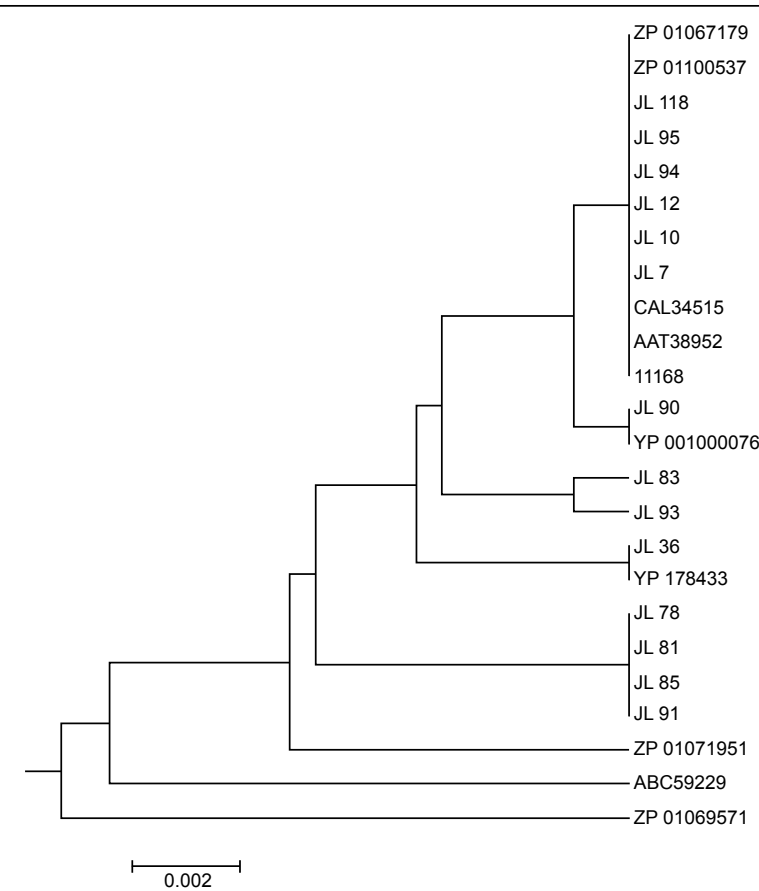

Figure 1: Phylogenetic relationship of $\mathrm{CmeC}$ from diverse $C$. jejuni strains. The amino acid based dendrogram was constructed by neighbor-joining methods in MEGA 4.0. The strains with 'JL' prefix were used for cmeC gene amplification and sequencing in this study and the complete cmeC sequences of these strains have been deposited in GenBank. The CmeC sequences of other strains are the public database NCBI (http://www.ncbi. nlm.nih.gov/)

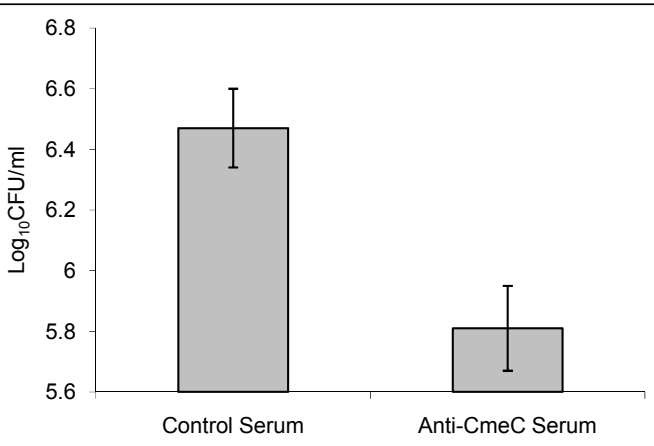

Figure 2: Growth responses of $C$. jejuni 81-176 to anti-CmeC serum and control serum (pre immune serum). The log-phase culture of 81-176 was diluted to approximately $2 \times 10^{6} \mathrm{cfu} / \mathrm{ml}$ in $\mathrm{MH}$ broth containing sublethal concentrations of cholic acid $(2 \mathrm{mg} / \mathrm{ml})$. Anti-CmeC and control sera were added to cells with 1:10 dilution and cells were incubated for 6 hours at $42^{\circ} \mathrm{C}$ under microaerophilic conditions. Samples were diluted in $\mathrm{MH}$ broth and plated on $\mathrm{MH}$ agar plates to determine bacterial viability. Each bar represents the mean value obtained from triplicate assays.

Microtiter plates (Nunc-Immuno Plate, Thermo Fisher scientific, Fairlawn, NJ) were coated with $100 \mu \mathrm{l}$ of $\mathrm{rCmeC} \mathrm{(30} \mathrm{ng/well)} \mathrm{in} \mathrm{coating}$ buffer (ammonium acetate plus ammonium carbonate [pH 8.2]) overnight at room temperature. For vaccination Trial 1 , to examine the levels of circulating $\mathrm{CmeC}$ specific $\operatorname{IgA}$ and $\lg \mathrm{G}$ antibodies and mucosal IgA antibodies, chicken serum and intestinal lavage samples were routinely diluted $1: 100$ and $1: 4$, respectively. For vaccination Trial 2, chicken intestinal lavage samples were routinely diluted 1:4 for ELISA test while each serum sample was serially diluted up to 4,096-fold for ELISA analysis. Three negative serum samples from individual chickens without receiving any treatment were used in each ELISA plate as background control. End point titer was defined as the last dilution at which the optical density of sample wells exceeded the mean optical density of three control wells plus $3 \times$ standard deviation of $\mathrm{OD}_{405 \mathrm{~nm}}$. Titer was expressed as the reciprocal of the end point dilution $\log _{2}$.

\section{Statistical analysis}

Differences in serum and intestinal lavage $\mathrm{OD}_{405 \mathrm{~nm}}$ readings or antibody titers among treatment groups were analyzed by least square analysis of covariance with the data at day 0 postimmunization as the covariant; main effects were day of sample collection and treatment. Comparison of lavage $\mathrm{OD}_{405 n \mathrm{~m}}$ readings or antibody titers within treatment groups across time were tested by ANOVA. Two-way ANOVA followed by Least significant difference (LSD) test were used to assess significance of difference among percentages and shedding levels of the groups at each sampling point. Levels of significance for $P$ value are 5\% (0.05). All statistical analyses were performed using SAS software (v9.03, SAS Institute Inc., Cary, NC).

\section{Results}

\section{CmeC is highly conserved in $C$. jejuni}

The full-length $\mathrm{CmeC}$ genes from 14 C. jejuni strains were PCR amplified and sequenced in this study. The new sequences were translated into deduced protein sequence and aligned with the $\mathrm{CmeC}$ sequences from $10 \mathrm{C}$. jejuni strains deposited in the NCBI public database (http://www.ncbi.nlm.nih.gov/sutils/genom_table.cgi). As shown in Figure 1, amino acid sequences of $\mathrm{CmeC}$ displayed a high level of homology ( $97.3 \%$ to $100 \%$ aa identity) among $C$. jejuni strains
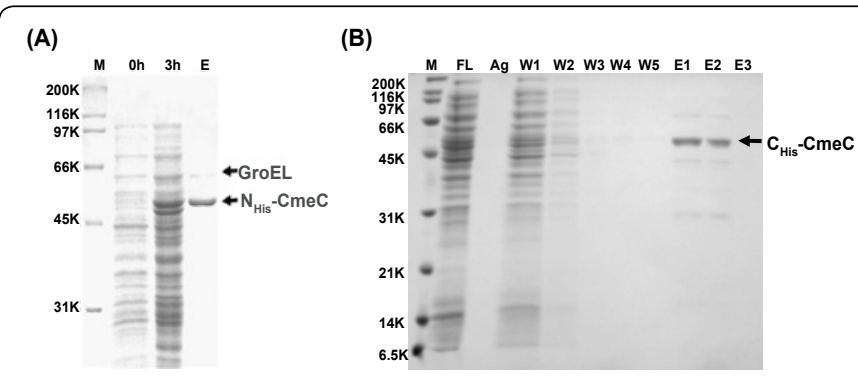

(C)

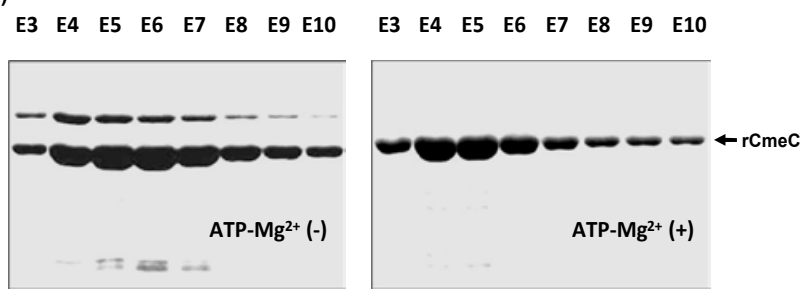

Figure 3: SDS-PAGE analysis of $\mathrm{rCmeC}$ production and purification from E. coli. (A) Expression and purification of $\mathrm{N}$ - terminal His-tagged $\mathrm{rCmeC}$. $\mathrm{M}$, standard molecular mass markers (Bio-Rad); $0 \mathrm{~h}$ and $3 \mathrm{~h}$, noninduced and $3 \mathrm{~h}$-induced whole-cell lysate, respectively; $\mathrm{E}$, eluted $\mathrm{rCmeC}$ fraction using Ni-NTA affinity chromatography (Qiagen). The putative GroEL, an $E$. coli molecule chaperone, was consistently co-purified with $\mathrm{rCmeC}$ using the standard protocol. (B) Expression and purification of C-terminal Histagged rCmeC. FL: flow through; Ag, Ni-NTA agarose after elution; W1-W5, washing fractions; E1-E3, elution fractions. (C) Efficient removal of GroEL contaminant by ATP-Mg ${ }^{2+}$ treatment. Eluted fractions (E3 to E10) during $\mathrm{Ni}$ NTA purification with (right panel) or without (left panel) addition of $5 \mathrm{mM}$ of ATP- $\mathrm{Mg}^{2+}$ were subjected to SDS-PAGE analysis. 
(A)

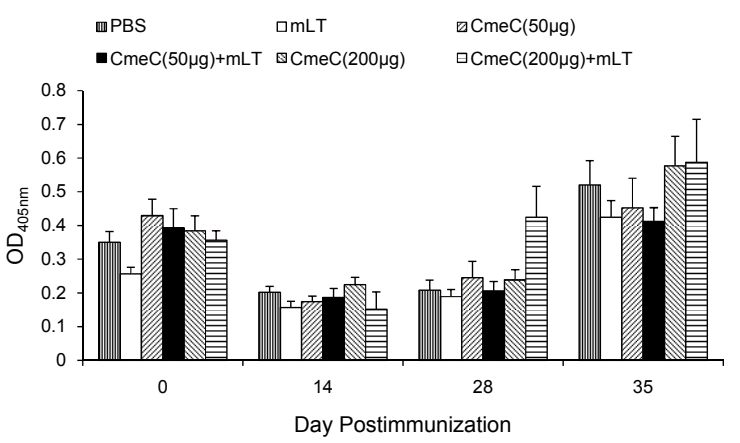

(B)

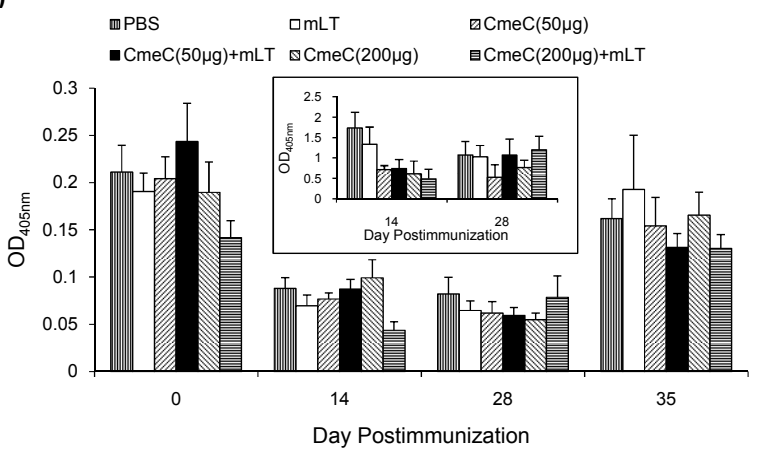

Figure 4: Chicken immunological responses to $\mathrm{rCmeC}$ vaccination (Trial 1). (A) Serum IgG response elicited by $\mathrm{rCmeC}$ subunit vaccination in broiler chickens. All chicken serum samples were diluted 1:100 for indirect ELISA. (B) Serum $\lg A$ and intestinal $\lg A$ (the embedded figure) levels in chickens vaccinated with $\mathrm{rCmeC}$. The ELISA procedure is detailed in Materials and Methods. Each bar is the average of OD readings from 10 to 20 individual serum samples or 5 individual intestinal lavage samples with standard error indicated by error bar.

with diverse backgrounds, suggesting that $\mathrm{CmeC}$ is highly conserved in $C$. jejuni. The secondary structure prediction and homologous modeling of $\mathrm{CmeC}$ using NodT (1wp1.pdb) as a template showed that the monomer of $\mathrm{CmeC}$ has 4 transmembrane beta sheets (data not shown); three such $\mathrm{CmeC}$ monomers may interact with each other and form a functional porin with 12-stranded transmembrane beta barrel to export substrates, as what has been shown for TolC, the CmeC homolog in E. coli [22].

\section{CmeC peptide antibodies enhanced susceptibility of $C$. jejuni} to bile salt

Supplementation of anti-CmeC serum in $\mathrm{MH}$ broth containing a sublethal concentration of cholic acid resulted in moderate but significant growth reduction $\left(\sim 0.6 \log _{10}\right.$ unit, $P<0.05$, Figure 2$)$ when compared to growth in the presence of control serum (preimmune serum, negative for $\mathrm{CmeC}$ ). This finding suggests that anti$\mathrm{CmeC}$ antibodies specifically bind to surface-exposed $\mathrm{CmeC}$, inhibit the function of CmeABC efflux pump, and increases the susceptibility of $C$.jejuni to bile salt.

\section{Production of high purity His-tagged $\mathrm{rCmeC}$}

The anti-CmeC serum used in above in vitro immune protection assay was directed against a portion of $\mathrm{CmeC}$ (aa 41 to 248) [11] and may not contain all protective epitopes of $\mathrm{CmeC}$. To better study immune protection conferred by $\mathrm{CmeC}$ vaccination in this study, two $E$. coli constructs were constructed for producing full-length rCmeC proteins. Both N-terminal (Figure 3A) and C-terminal Histagged $\mathrm{rCmeC}$ (Figure 3B) were successfully produced in E. coli and purified by Ni-NTA agarose affinity column. However, the yield of C-terminal His-tagged $\mathrm{rCmeC}$ was significantly lower than that of $\mathrm{N}$-terminal His-tagged $\mathrm{rCmeC}$; Approximately $1.5 \mathrm{mg}$ of $\mathrm{N}$ terminal His-tagged $\mathrm{rCmeC}$ was consistently purified from $100 \mathrm{ml}$ of induced culture using $1 \mathrm{ml}$ of Ni-NTA resin. Thus, to produce large amount of $\mathrm{rCmeC}$ for vaccination studies, we then focused on optimization of purification for $\mathrm{N}$-terminal His-tagged $\mathrm{rCmeC}$. As shown in Figure $3 \mathrm{~A}$, a protein band with approximate $\mathrm{MW}$ of $60 \mathrm{kDa}$ was consistently co-purified with $\mathrm{rCmeC}$ when following the standard protocol. Based on the molecular weight of this co-purified protein, it is likely this contaminated protein is E. coli molecule chaperone GroEL [19]. Since it has been reported that including $\mathrm{Mg}^{2+}$-ATP in washing buffer could efficiently remove the GroEL contamination [23], the purification procedure was modified to improve the purity of the extracted rCmeC. As shown in Figure 3C, addition of $5 \mathrm{mM}$ of $\mathrm{Mg}^{2+}$-ATP in lysis buffer completely removed contaminated bands, resulting in eluents containing $\mathrm{rCmeC}$ with high purity. Large quantities of highpurity $\mathrm{rCmeC}$ was obtained using the modified procedure for the vaccination and ELISA in this study.

\section{Immunological responses and protective efficacy following CmeC vaccination}

In Trial 1 , oral vaccination of chickens with or without $\mathrm{mLT}$ adjuvant did not significantly $(P>0.05)$ enhance $\operatorname{IgG}$ and $\operatorname{IgA}$ levels in serum and in intestinal lavage at different days postimmunization (Figure 4). Despite the variations observed among individual chickens, chickens in group $6(200 \mu \mathrm{g} \mathrm{rCmeC}+\mathrm{mLT})$ at $28 \mathrm{~d}$ postimmunization

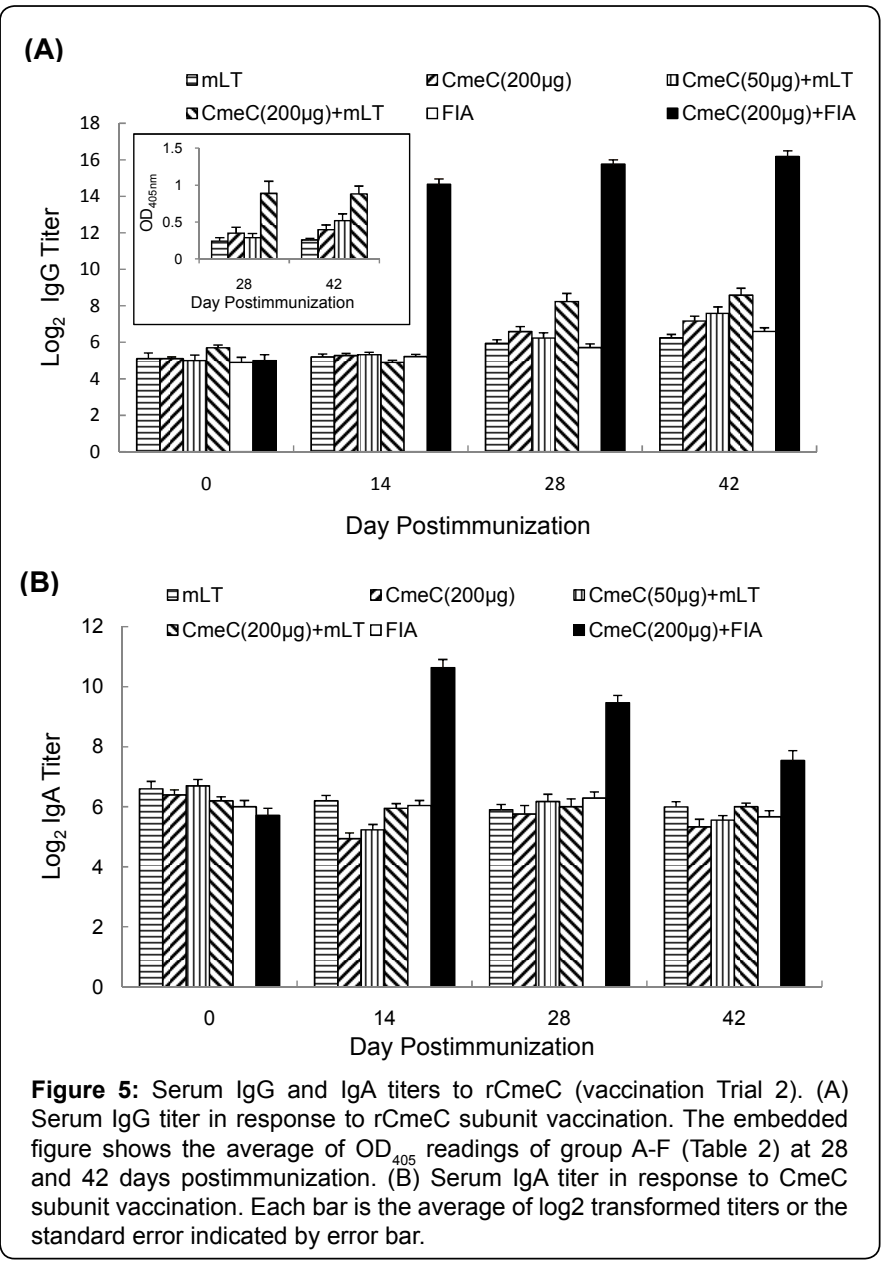


Citation: Zeng X, Xu F, Lin J (2010) Development and Evaluation of CmeC Subunit Vaccine against Campylobacter jejuni. J Vaccines Vaccin 1: 112. doi:10.4172/2157-7560.1000112

Page 6 of 8

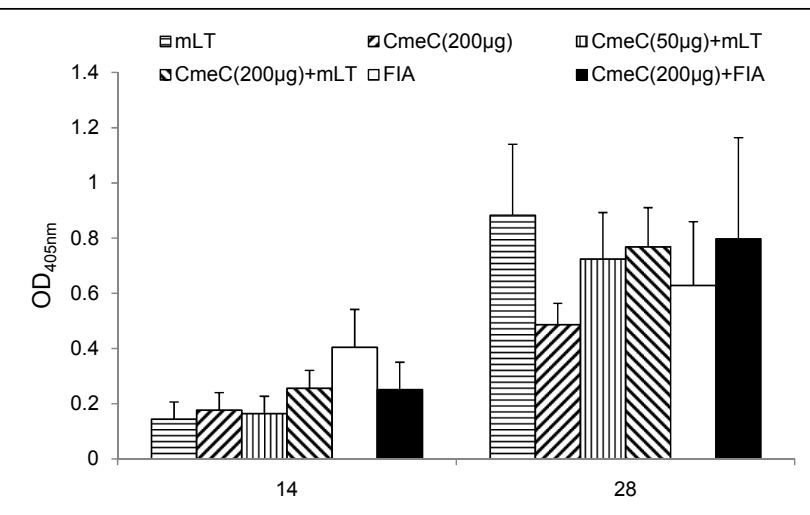

Day Postimmunization

Figure 6: Intestinal mucosal IgA titers to $\mathrm{CmeC}$ (vaccination Trial 2). Each bar is the average of $O_{405 \mathrm{~m}}$ readings from 5 individual intestinal lavage samples with standard error indicated by error bar.

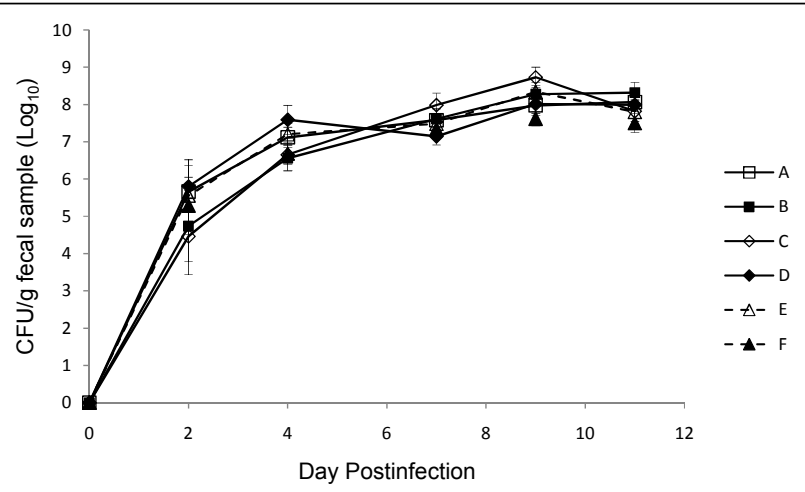

Figure 7: Campylobacter colonization levels after challenging CmeCvaccinated chickens with $C$. jejuni NCTC 11168 (Trial 2). The letter for each type of point corresponds to the group described in Table 3. Each point represents mean $\log _{10}$ CFU/g feces \pm the standard deviation in each group.

showed consistently and slightly higher serum $\lg G$ level $(P=$ 0.57) than PBS control group. Interestingly, all groups displayed relatively higher $\operatorname{Ig} G$ and $\operatorname{IgA}$ levels immediately prior to primary CmeC immunization (Figure 4), likely due to the effect of maternal antibodies [21,24]. Consistent with the patterns of these antibody responses, challenge of chickens with NCTC 11168 at 28 days after primary $\mathrm{CmeC}$ vaccination did not show a significant difference of colonization among groups $(P>0.05)$ (Data not shown). All chickens in each group were quickly colonized by $C$. jejuni NCTC 11168 two days after challenge with an average shedding level of $\sim 10^{7} \mathrm{CFU} / \mathrm{g}$ feces, likely due to the high dose of inoculums used for challenge (10 $\mathrm{CFU} / \mathrm{bird})$.

In Trial 2, oral immunization of chickens with a higher dose of CmeC $(200 \mu \mathrm{g})$ and mucosal adjuvant $(70 \mu \mathrm{g})$ significantly $(P<0.05)$ elevated the IgG titer than those in the control groups (receiving $\mathrm{mLT}$

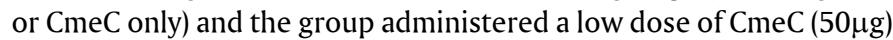
plus $\mathrm{mLT}$ at 28 and $42 \mathrm{~d}$ postimmunization (Figure $5 \mathrm{~A}$ ). As shown in the embedded figure in Figure $5 \mathrm{~A}$, the $\mathrm{OD}_{405 \mathrm{~nm}}$ values for higher dose of $\mathrm{CmeC}(200 \mu \mathrm{g})$ together with $70 \mu \mathrm{g}$ of $\mathrm{mLT}$ were $0.89 \pm 0.16$ and $0.88 \pm 0.10$ at $28 \mathrm{~d}$ and $42 \mathrm{~d}$ postimmunization, respectively, which are significantly higher $(P<0.05)$ than the mean $\mathrm{OD}_{405 \mathrm{~nm}}$ values from other groups. However, serum IgA titers were similar among the groups that received oral vaccination (Figure 5B). Subcutaneous vaccination of chickens with $\mathrm{CmeC}$ together with Freund's incomplete adjuvant dramatically elevated serum $\operatorname{IgG}$ and $\operatorname{Ig} A$ titers $(P<0.05)$ at
14,28 , and $42 \mathrm{~d}$ postimmunization compared with the control group vaccinated with Freund's incomplete adjuvant only. However, CmeC vaccination did not trigger significantly higher level of intestinal secretory $\operatorname{IgA}$ against $\mathrm{rCmeC}$, regardless of vaccination route and dosage (Figure 6). Consistent with the lack of significantly induced local IgA response to $\mathrm{CmeC}, \mathrm{CmeC}$ vaccination in Trial 2 did not significantly reduce $C$. jejuni colonization in the intestine (Figure 7).

\section{Discussion}

Previous studies have reported that $\mathrm{CmeC}$ is prevalent in $C$. jejuni, is induced and immunogenic in vivo, and is essential for $C$. jejuni colonization [10-13]. These findings suggest that $\mathrm{CmeC}$ is a promising subunit vaccine candidate against $C$. jejuni colonization in the intestine. The in vitro studies in this project provided further compelling evidence that $\mathrm{CmeC}$ is an attractive candidate for $C$. jejuni vaccine development. Alignment of complete $\mathrm{CmeC}$ sequences from diverse strains demonstrated that $\mathrm{CmeC}$ is highly conserved in C. jejuni (Figure 1). This finding is consistent with a recent report in which a small portion of $\mathrm{cmeC}$ was PCR amplified from different Campylobacter strains for sequencing [25]. It is likely that $\mathrm{CmeC}$ displayed variation between different Campylobacter species, such as $C$. jejuni and $C$. coli, because alignment of partial $\mathrm{CmeC}$ sequence showed only $83 \%$ aa identity between $C$. jejuni and $C$. coli [25]. However, sequence analysis of full length $\mathrm{CmeC}$ in this study clearly indicated that the genetic variation within $C$. jejuni, which is responsible for $\sim 90 \%$ of human campylobacteriosis [1], is extremely limited. This evidence together with in vitro inhibitory effect of $\mathrm{CmeC}$ peptide antiserum on the function of CmeABC efflux pump (Figure 2) provides a strong rationale to develop $\mathrm{CmeC}$-based subunit vaccine.

To overcome the potential limitations of using partial $\mathrm{CmeC}$ peptide for vaccination studies, such as lack of enough protective epitopes, the full-length $\mathrm{rCmeC}$ proteins were produced and purified in this study. Expression and purification of recombinant membrane proteins in E. coli system are always coupled with problems such as low yield, insolubility, unfolding or misfolding, co-purification of contaminations, proteolytic degradation, and less biological activity. In the past decade, extensive efforts have been placed on the modification of promoter, design of fusion with tags, refolding after purification, chaperone co expression/knockout, and protease gene knockout [26-28]. In this study, the $\mathrm{N}$-terminal His tagged $\mathrm{rCmeC}$ is located in cytoplasm due to removal of the 19-aa signal peptide. Thus, cytoplasmic $\mathrm{rCmeC}$ proteins which have hydrophobic transmembrane domains are likely to attract/arrest the chaperones [28]. During our purification of $\mathrm{rCmeC}$, we consistently observed the co-purification of a protein with molecular weight of $\sim 60 \mathrm{kDa}$ together with the target $\mathrm{rCmeC}$ band (Figure $3 \mathrm{~A}$ and $3 \mathrm{C}$ ), suggesting that the GroEL $(60 \mathrm{kDa})$-GroES $(10 \mathrm{kDa})$ chaperone system in E. coli $[19,26]$ may bind newly synthesized $\mathrm{rCmeC}$. Since the binding of ATP can trigger the turnover of substrates in GroEL-GroES systems by reducing the affinity of GroEL-GroES with substrates [23], $5 \mathrm{mM}$ of $\mathrm{Mg}^{2+}$-ATP was added into the lysate to facilitate the disassociation of $\mathrm{rCmeC}$ from GroEL-GroES. This single and simple modification increased the purity of extracted $\mathrm{rCmeC}$ without affecting yield (Figure 3C). It is also important to mention that the detergent Empigen $\mathrm{BB}$ was used to facilitate solubilization of $\mathrm{rCmeC}$ during purification in this study because Empigen $\mathrm{BB}$ is a mild zwitterionic detergent and is known for its ability to preserve the antigenicity and functional activity of isolated proteins [29]. Together, the $\mathrm{rCmeC}$ protein with high purity has been obtained in this study for various research efforts, such as vaccination described in this study and crystallization in the future studies. 
Citation: Zeng X, Xu F, Lin J (2010) Development and Evaluation of CmeC Subunit Vaccine against Campylobacter jejuni. J Vaccines Vaccin 1: 112. doi:10.4172/2157-7560.1000112

Page 7 of 8

We have established a chicken model of $C$. jejuni infection and used this model to study the critical role of $\mathrm{CmeC}$ in colonization of $C$. jejuni [12]. We chose to use chickens for vaccination and $C$. jejuni challenge studies primarily for the following reasons. First, chickens are a natural host for $C$. jejuni. The high susceptibility of chicken to Campylobacter colonization, the ease of handling, and the low cost of chickens have provided an ideal model system to study the colonization and immunogenicity of $C$. jejuni in the host $[8,12,15,16,30]$. Second, newly hatched chickens are always Campylobacter-free, providing a clean and economical host for evaluating Campylobacter colonization [21]. Third, to date, there are no appropriate disease models for $C$. jejuni infection; a recently developed non-human primate model system may partly mimic symptoms caused by $C$. jejuni infection in humans [31]. Although $C$. jejuni does not cause clinical disease in chickens (an infection model, not a disease model), chickens are a sufficient and powerful colonization model to evaluate adaptation and survival of Campylobacter in response to harsh in vivo conditions (e.g. bile salts relevant to this study). Fourth, the heat labile enterotoxin (LT) has been used as a mucosal adjuvant with chicken oral vaccines [32,33], which provides a rationale for us to use $\mathrm{mLT}$ together with the $\mathrm{CmeC}$ oral vaccine in chicken model system described in this study. Finally, poultry is considered a major source for $C$. jejuni infection in humans [7]. Reduction of Campylobacter in poultry by vaccination of chickens will reduce the risk of exposure by humans who consume poultry products. Therefore, findings from chicken studies are also directly relevant to food safety and public health.

Oral vaccination of chickens, other animals as well as humans with subunit vaccine in conjunction with adjuvant LT or mLT have been demonstrated to induce protective immunity [32-35]. Unexpectedly, oral vaccination of $\mathrm{CmeC}$ with $\mathrm{mLT}$ in two trials performed in this study did not trigger significant local immune response to $\mathrm{CmeC}$ and thus failed to confer protection of chickens against $C$. jejuni colonization. However, the findings from this study provided useful information for future development and evaluation of $\mathrm{CmeC}$ subunit vaccine using the chicken as a model. First, white leghorn appears a better animal host than broiler for vaccination evaluation, primarily due to its slow growth rate, which makes animal handling easier and also allows us to initiate late primary vaccination (e.g. 3 wk of age) when Campylobacter specific maternal antibodies decrease to a low level. Second, although dramatic systemic immune response to $\mathrm{CmeC}$ was induced using the subcutaneous vaccination route in this study, the elevated antibody titers in serum did not result in increased levels of intestinal antibodies, which suggests that vaccination via mucosal route, such as oral vaccine, should be used to induce a strong gut mucosal immune response to control Campylobacter colonization. Third, development of an oral subunit vaccine faces a common difficulty: weak immunogenicity due to the choice of adjuvant and antigen degradation in the GI tract. Previous studies have shown that Campylobacter-specific secretory and serum IgA antibodies correlate with protection against Campylobacter infection [36]. In this study, sufficient levels of $\mathrm{CmeC}$-specific antibodies, particularly intestinal $\operatorname{IgA}$, were not reached for protection against $C$. jejuni infection. Given that the $\mathrm{mLT}$ used in this study has been shown to be effective with oral vaccines in different animals including chickens at low dose (e.g. $5 \mu$ g single dose for pig) [35], it is unlikely that the mLT fails to stimulate immune response if it reaches chicken gut together with $\mathrm{rCmeC}$. We speculate that orally administered $\mathrm{rCmeC}$ and/or $\mathrm{mLT}$ may be absorbed to the upper gastrointestinal tract and significantly degraded in the intestine before it can prime the host immune system. To solve this problem, appropriate delivery systems may be explored to enhance mucosal immune response. For example, encapsulation of $\mathrm{rCmeC}$ using the chitosan microsphere, an effective adjuvant/carrier system $[37,38]$, may be a promising approach to deliver $\mathrm{rCmeC}$ to the target site and trigger a strong local intestinal mucosal immune response. In addition, identification of protective epitopes of $\mathrm{CmeC}$ followed by construction of live Salmonella-vectored vaccine is also a promising approach for persistent deliver specific protective epitopes to induce local mucosal response [39]. Elucidation of the structure of $\mathrm{CmeC}$ by taking advantage of the high purity $\mathrm{rCmeC}$ obtained from this study for crystallization will facilitate construction of a Salmonella live vaccine expressing specific surface region(s) of $\mathrm{CmeC}$. These hypotheses will be tested in future studies.

Successful development of $\mathrm{CmeC}$-based vaccine may lead to a novel bifunctional vaccine that not only prevents Campylobacter colonization but also combats antibiotic resistance in $C$. jejuni. It has been demonstrated that inhibition of multidrug efflux pumps by efflux pump inhibitors (EPIs) is an effective approach to improve the clinical performance of antibiotics [40-42]. We also observed that inhibition of CmeABC pump by an efflux pump inhibitor increased susceptibilities of $C$. jejuni to various antibiotics and significantly reduced the frequency of emergence of macrolide resistant $\mathrm{C}$. jejuni $[15,16]$. However, several key issues (e.g. toxicity, in vivo stability, production cost) should be well addressed before EPIs can be used clinically and accepted by medical community. Given the significant role of $\mathrm{CmeC}$ in multidrug resistance, we hypothesize that $\mathrm{CmeC}$ antibodies may function similarly as EPI by targeting an essential component of CmeABC pump and immunization of chickens with $\mathrm{CmeC}$ vaccine may enhance the activity of clinical antibiotics against C. jejuni. Compared to EPIs, the CmeC vaccine may be a more realistic approach to potentiate the activity of clinical antibiotics against Campylobacter. Thus, CmeC may also represent a novel vaccine that can enhance the efficacy of clinical antibiotics and even reduce the frequency of in vivo emergence of antibiotic resistant $C$. jejuni. This hypothesis needs to be determined after optimization of $\mathrm{CmeC}$ vaccination regimen in the future.

\section{Acknowledgments}

We are grateful to Dr. John D. Clements (Tulane University Medical Center) for kindly providing the mucosal adjuvant $\mathrm{mLT}-\mathrm{R} 192 \mathrm{G}$ as a gift for the vaccination studies in this paper. We thank Ky Van Hoang, Andree A. Hunkapiller, and Ad'lynn Martinez for technical support. This study was supported by grant 1R21AI07255101 from NIH.

\section{References}

1. Olson CK, Ethelberg S, Pelt WV, Tauxe RV (2008) Epidemiology of Campylobacter jejuni infections in industrialized countries. p. 163-189. In Nachamkin I, Szymanski CM and Blaser MJ (ed.), Campylobacter, 3rd ed., ASM Press, Washington, DC.

2. Rees JH, Soudain SE, Gregson NA, Hughes RA (1995) Campylobacter jejun Infection and Guillain-Barre syndrome. N Engl J Med 333(21): 1374-1379.

3. Nachamkin I, Allos BM, Ho T (1998) Campylobacter species and Guillain-Barre Syndrome. Clin Microbiol Rev 11: 555-567.

4. Young KT, Davis LM, Dirita VJ (2007) Campylobacter jejuni: molecular biology and pathogenesis. Nat Rev Microbiol 5: 665-679.

5. Engberg J Aarestrup FM, Taylor DE Gerner-Smidt P, Nachamkin I (2001) Quinolone and macrolide resistance in Campylobacter jejuni and C. coli resistance mechanisms and trends in human isolates. Emerg Infect Dis 7: 24 34.

6. Projan SJ (2003) Why is big Pharma getting out of antibacterial drug discovery? Curr Opin Microbiol 6: 427-430.

7. Lin J (2009) Novel approaches for Campylobacter control in poultry. Foodborne Pathog Dis 6: 755-765. 
Citation: Zeng X, Xu F, Lin J (2010) Development and Evaluation of CmeC Subunit Vaccine against Campylobacter jejuni. J Vaccines Vaccin 1: 112. doi:10.4172/2157-7560.1000112

Page 8 of 8

8. de Zoete MR, van Putten JP, Wagenaar JA (2007) Vaccination of chickens against Campylobacter. Vaccine 25: 5548-557.

9. Jagusztyn-Krynicka EK, Laniewski P, Wyszynska A (2009) Update on Campylobacter jejuni vaccine development for preventing human campylobacteriosis. Expert Rev Vaccines 8: 625-645.

10. Lin J, Akiba M, Sahin O, Zhang Q (2005) CmeR functions as a transcriptional repressor for the multidrug efflux pump CmeABC in Campylobacter jejuni. Antimicrob Agents Chemother 49: 1067-1075.

11. Lin J, Michel LO, Zhang Q (2002) CmeABC functions as a multidrug efflux system in Campylobacter0 jejuni. Antimicrob Agents Chemother 46: 21242131.

12. Lin J, Sahin O, Michel LO, Zhang Q (2003) Critical role of multidrug efflux pump CmeABC in bile resistance and in vivo colonization of Campylobacter jejuni. Infect Immun 71: 4250-4259.

13. Lin J, Cagliero C, Guo B, Barton YW, Maurel MC, et al. (2005) Bile salts modulate expression of the CmeABC multidrug efflux pump in Campylobacter jejuni. J Bacteriol 87: 7417-7424.

14. Stintzi A, Marlow D, Palyada K, Naikare H, Panciera R, et al. (2005) Use of genome5 wide expression profiling and mutagenesis to study the intestinal lifestyle of Campylobacter jejuni. Infect Immun 73: 1797-1810.

15. Martinez A, Lin J (2006) Effect of an efflux pump inhibitor on the function of the multidrug efflux pump CmeABC and antimicrobial resistance in Campylobacter. Foodborne Pathog Dis 3: 393-402.

16. Lin J, Martinez A (2006) Effect of efflux pump inhibitors on bile resistance and in vivo colonization of Campylobacter jejuni. J Antimicrob Chemother 58: 966972

17. Xu F, Zeng X, Haigh RD, Ketley JM, Lin J (2010) Identification and Characterization of a New Ferric Enterobactin Receptor, CfrB, in Campylobacter. J Bacteriol 192: 4425-4435

18. Akiba M, Lin J, Barton YW, Zhang Q (2006) Interaction of CmeABC and CmeDEF in conferring antimicrobial resistance and maintaining cell viability in Campylobacter jejuni. J Antimicrob Chemother 57: 52-60.

19. Graslund S, Nordlund P, Weigelt J, Hallberg BM, Bray J, et al. (2008) Protein production and purification. Nat Methods 5: 135-146.

20. Wyszynska A, Raczko A, Lis M, Jagusztyn-Krynicka EK (2004) Ora immunization of chickens with avirulent Salmonella vaccine strain carrying $C$. jejuni 72Dz/92 cjaA gene lelicits specific humoral immune response associated with protection against challenge with wild-type Campylobacter. Vaccine 22: 1379-1389.

21. Sahin O, Zhang Q, Meitzler JC, Harr BS, Morishita TY, et al. (2001) Prevalence antigenic specificity, and bactericidal activity of poultry anti-Campylobacter maternal antibodies. Appl Environ Microbiol 67: 3951-3957.

22. Andersen C, Koronakis E, Bokma E, Eswaran J, Humphreys D, et al. (2002) Transition to the open state of the TolC periplasmic tunnel entrance. Proc Nat Acad Sci U S A 99: 11103-11108.

23. Harjes S, Scheidig A, Bayer P (2004) Expression, purification and crystallization of human 3'-phosphoadenosine-5'-phosphosulfate synthetase 1. Acta Crystallogr D Biol Crystallogr 60: 350-352.

24. Sahin O, Luo N, Huang S, Zhang Q (2003) Effect of Campylobacter-specific maternal antibodies on Campylobacter jejuni colonization in young chickens. Appl Environ Microbiol 69: 5372-5379.

25. Fakhr MK, Logue CM (2007) Sequence variation in the outer membrane protein37 encoding gene $\mathrm{cmeC}$, conferring multidrug resistance among Campylobacter jejuni and Campylobacter coli strains isolated from different hosts. J Clin Microbiol 45: 3381-3383.

26. de Marco A (2007) Protocol for preparing proteins with improved solubility by co40 expressing with molecular chaperones in Escherichia coli. Nat Protoc 2: 2632-2639.
27. de Marco A (2006) Two-step metal affinity purification of double-tagged (NusAHis6) fusion proteins. Nat Protoc 1: 1538-1543.

28. de Marco A, Deuerling E, Mogk A, Tomoyasu T, Bukau B (2007) Chaperonebased procedure to increase yields of soluble recombinant proteins produced in E. coli. BMC Biotechnol 12: 7-32.

29. Zhang Q, Meitzler JC, Huang S, Morishita T (2000) Sequence polymorphism predicted secondary structures, and surface-exposed conformational epitopes of Campylobacter major outer membrane protein. Infect Immun 68: 5679-5689.

30. Conlan AJ, Coward C, Grant AJ, Maskell DJ, Gog JR (2007) Campylobacter jejuni colonization and transmission in broiler chickens: a modelling perspective. J R Soc Interface 4: 819-829.

31. Monteiro MA, Baqar S, Hall ER, Chen YH, Porter CK, et al. (2009) Capsule polysaccharide conjugate vaccine against diarrheal disease caused by Campylobacter jejuni. Infect Immun 77: 1128-1136.

32. Rice BE, Rollins DM, Mallinson ET, Carr L, Joseph SW (1997) Campylobacte jejuni in broiler chickens: colonization and humoral immunity following ora vaccination and experimental infection. Vaccine 15: 1922-1932.

33. Wilkinson J, Rood D, Minior D, Guillard K, Darre M, et al. (2003) Immune response to a mucosally administered aflatoxin B1 vaccine. Poult Sci 82: 15651572.

34. Lapa JA, Sincock SA, Ananthakrishnan M, Porter CK, Cassels FJ, et al. (2008) Randomized clinical trial assessing the safety and immunogenicity of oral microencapsulated enterotoxigenic Escherichia coli surface antigen 6 with or without heat-labile enterotoxin with mutation R192G. Clin Vaccine Immunol 15: 1222-1228.

35. Yuan L, losef C, Azevedo MS, Kim Y, Qian Y, et al. (2001) Protective immunity and antibody-secreting cell responses elicited by combined oral attenuated Wa human rotavirus and intranasal Wa 2/6-VLPs with mutant Escherichia coli heatlabile toxin in gnotobiotic pigs. J Virol 75: 9229-6238.

36. Tribble DR, Baqar S, Thompson SA (2008) Development of a Human Vaccine Pages: 429-444. In I. Nachamkin, C. M. Szymanski, and M. J. Blaser (ed.) Campylobacter, 3rd ed. ASM Press, Washington, DC.

37. Sinha VR, Singla AK, Wadhawan S, Kaushik R, Kumria R, et al. (2004) Chitosan microspheres as a potential carrier for drugs. Int J Pharm 274: 1-33.

38. Arca HC, Gunbeyaz M, Senel S (2009) Chitosan-based systems for the delivery of Vaccine antigens. Expert Rev Vaccines 8: 937-953.

39. Kwon YM, Cox MM, Calhoun LN (2007) Salmonella-based vaccines for infectious diseases. Expert Rev Vaccines 6: 147-152.

40. Lomovskaya O, Warren MS, Lee A, Galazzo J, Fronko R, et al. (2001) Identification and characterization of inhibitors of multidrug resistance efflux pumps in Pseudomonas 25 aeruginosa: novel agents for combination therapy. Antimicrob Agents Chemother 45: 105-116.

41. Lomovskaya O and Watkins W (2001) Inhibition of efflux pumps as a nove approach to combat drug resistance in bacteria. J Mol Microbiol Biotechnol 3 . 225-2236.

42. Ryan BM, Dougherty TJ, Beaulieu D, Chuang J, Dougherty BA, et al. (2001) Efflux in bacteria: what do we really know about it? Expert Opin Investig Drugs 10: $1409-1422$.

43. Huang S, Luangtongkum T, Morishita TY, Zhang Q, (2005) Molecular typing of Campylobacter strains using the $\mathrm{cmp}$ gene encoding the major outer membrane protein. Foodborne Pathog Dis 2: 12-23.

44. Zeng X, Xu F and Lin J (2009) Molecular, antigenic, and functional characteristics of ferric enterobactin receptor CfrA in Campylobacter jejuni. Infect Immun 77 : 5437-5448.

45. Parkhill J, Wren BW, Mungall K, Ketley JM, Churcher C, et al. (2000) The genome sequence of the food-borne pathogen Campylobacter jejuni reveals hypervariable sequences. Nature 403: 665-668.

46. Black RE, Levine MM, Clements ML, Hughes TP, Blaser MJ (1988) Experimenta pylobacter jejuni infection in humans. J Infect Dis 157: 472-479. 